\title{
Comparison of three early biomarkers for acute kidney injury after cardiac surgery under cardiopulmonary bypass
}

Takahiro Moriyama1*, Shintaro Hagihara', Toko Shiramomo', Misaki Nagaoka², Shohei Iwakawa and Yuichi Kanmura ${ }^{1}$

\begin{abstract}
Background: Acute kidney injury (AKI) is a serious complication after cardiac surgery, being associated with a high mortality. We assessed three urinary biomarkers, L-type fatty acid-binding protein (L-FABP), neutrophil gelatinase-associated lipocalin (NGAL), and angiotensinogen, which are elevated through different mechanisms, and investigated which of these biomarkers was the earliest and most useful indicator of AKI after cardiac surgery.

Methods: This study was a prospective observational study conducted at a single-institution university hospital. All patients were adults aged under 80 years who underwent cardiac surgery with cardiopulmonary bypass between November 2013 and January 2015. Perioperatively, urine samples were obtained from all patients at five points. Based on AKI criteria, patients were divided into two groups: AKI group $(n=11)$ and non-AKI group $(n=39)$, according to postoperative serum creatinine $(\mathrm{Cr})$ levels.

Results: Urinary L-FABP, NGAL, angiotensinogen, and $\mathrm{Cr}$ were measured perioperatively. L-FABP was significantly higher in the AKI group than in the non-AKI group at the end of surgery and $3 \mathrm{~h}$ after surgery. L-FABP levels were $601.5 \pm 341.7$ and $233.8 \pm 127.2 \mu \mathrm{g} / \mathrm{g} \mathrm{Cr}$ in the AKI and non-AKI groups, respectively. Three hours after surgery, NGAL levels were $950.5 \pm 827.9$ and $430.0 \pm 250.6 \mu \mathrm{g} / \mathrm{g} \mathrm{Cr}$ in the AKI and non-AKI groups, respectively, the level being significantly higher in the AKI group than in the non-AKI group. There were no significant differences in urinary angiotensinogen levels between the two groups at any time point.

Conclusions: We demonstrated the utility of L-FABP and NGAL, but not angiotensinogen in the early recognition of AKI. The problem of the different peak points among biomarkers needs to be resolved for discovery of a panel of biomarkers.
\end{abstract}

Keywords: Acute kidney injury, Cardiac surgery, L-type fatty acid-binding protein (L-FABP), Neutrophil gelatinase-associated lipocalin (NGAL), Urinary angiotensinogen

\section{Background}

Acute kidney injury (AKI) is one of the most common and serious complications after cardiac surgery, being associated with a high mortality [1]. Depending on the definition used for AKI, its incidence after cardiac surgery reportedly ranges from 5 to $30 \%[2,3]$. Traditionally, serum creatinine $(\mathrm{sCr})$ has been the main marker for the diagnosis of AKI. There are three criteria

\footnotetext{
* Correspondence: takmor@m3.kufm.kagoshima-u.ac.jp

${ }^{1}$ Department of Anesthesiology and Intensive Care, Kagoshima University

Hospital, Sakuragaoka 8-35-1, Kagoshima 46201, Japan

Full list of author information is available at the end of the article
}

for the diagnosis of AKI, termed the Risk, Injury, Failure, Loss of kidney function and End stage of kidney disease (RIFLE) criteria, Acute Kidney Injury Network (AKIN) criteria, and Kidney Disease: Improving Global Outcomes (KDIGO) criteria [4-6]. These criteria include increasing values and rates of increases of sCr. However, $\mathrm{sCr}$ is not an ideal biomarker for AKI after cardiac surgery, because it takes $24-48 \mathrm{~h}$ after renal damage for sCr levels to increase, and glomerular filtration rate (GFR) needs to reduce by approximately $75 \%$ before $\mathrm{sCr}$ increases to abnormal values [7]. Moreover, previous studies reported that even a small 
increase in $\mathrm{sCr}$ ( 0 to $0.5 \mathrm{mg} / \mathrm{dl})$ is associated with increasing mortality [3]. sCr also has limited sensitivity and specificity because of several factors such as age, sex, muscle mass, hypertension, and metabolism that can affect its value [8]. Further, renal function can be significantly impaired to an extent that requires renal replacement therapy despite normal $\mathrm{sCr}$ levels [9]. A previous study reported that $\mathrm{sCr}$ might underestimate the degree of renal failure, especially in elderly patients [10]. Therefore, the importance of earlier and more sensitive biomarkers for the diagnosis of AKI has been recently suggested.

Many urinary and plasma biomarkers for the detection of AKI have been investigated. AKI induced by different mechanisms, such as inflammation, reactive oxygen stress, and intrarenal renin-angiotensin systems, leads to secretion of various biomarkers into urine. In the present study, we assessed three urinary biomarkers, L-type fatty acid-binding protein (L-FABP), neutrophil gelatinase-associated lipocalin (NGAL), and urinary angiotensinogen, whose urinary concentrations are elevated through the different mechanisms. The purpose of this study was to investigate which of these biomarkers is the earliest and most useful indicator of AKI after cardiac surgery under cardiopulmonary bypass (CPB).

\section{Methods}

\section{Study design and patient population}

This prospective observational study was approved by the Ethics Committee of Kagoshima University Hospital and registered with the UMIN Clinical Trials Registry (UMIN 000012312) on November 18, 2013. The present study was conducted in accordance with the principles of the Declaration of Helsinki, and prior written informed consent was obtained from each patient. Fifty patients undergoing cardiac surgery under CPB were enrolled between November 2013 and January 2015. The exclusion criteria for participants in this study were emergency operation, low left ventricular function (ejection fraction $<40 \%$ ), elderly age (more than 80 years), and preoperative renal dysfunction $(\mathrm{sCr}$ level $>1.3 \mathrm{mg} / \mathrm{dl}$ ), all of which might directly increase the risk of occurrence of postoperative AKI.

\section{General management}

Patients were prepared according to the standard preoperative procedures after overnight fasting. Anesthesia was induced by injecting $0.08 \mathrm{mg} / \mathrm{kg}$ midazolam, $5 \mu \mathrm{g} / \mathrm{kg}$ fentanyl, and $0.6 \mathrm{mg} / \mathrm{kg}$ rocuronium bromide intravenously. Propofol was infused at the rate of $4-10 \mathrm{mg} / \mathrm{kg} / \mathrm{min}$ to maintain bispectral index (BIS; Aspect Medical Systems, Norwood, MA, USA) within the range of 40-60. Remifentanil was also administered as an infusion, the dose being maintained in the range of $0.1-0.5 \mu \mathrm{g} / \mathrm{kg} / \mathrm{min}$ depending on surgical invasiveness. After the induction of anesthesia, the lungs were ventilated to normocapnia and red blood cells were transfused to maintain a hematocrit of $25 \%$ or more.

\section{Measurements}

Urine samples were obtained at five points (before surgery (baseline), before and after $\mathrm{CPB}$, at the end of surgery, and $3 \mathrm{~h}$ after surgery). A 5-ml urine sample was collected at each point and stored at $-80{ }^{\circ} \mathrm{C}$ for later measurements of urinary NGAL, L-FABP, angiotensinogen, and creatinine. Moreover, $\mathrm{sCr}$ was measured preoperatively and on postoperative days 1, 2, and 3. Urinary L-FABP, NGAL, and angiotensinogen levels were determined using specific ELISA kits (CMIC Co. Ltd, Tokyo, Bioporto Diagnostics, Hellerup, Denmark, Japan, and IBL, Gunma, Japan, respectively) and were adjusted for urinary creatinine concentrations.

\section{Definition of AKI}

In the present study, we adopted KDIGO criteria to define AKI, according to which AKI after cardiac surgery is defined as an increase in $\mathrm{sCr}$ of $\geq 0.3 \mathrm{mg} / \mathrm{dl}$ or a percentage increase of $\geq 50 \%$ for $48 \mathrm{~h}$ after surgery [6]. Based on the KDIGO criteria, patients were divided into two groups: AKI group $(n=11)$ and non-AKI group $(n=39)$.

\section{Statistical analysis}

Data of patient characteristics and clinical outcomes are expressed as mean \pm standard deviations (SD). Patient background and outcome variables between the two groups were compared using Fisher's exact probability test or unpaired Student's $t$ tests using GraphPad Prism version 5. Data of AKI biomarkers are expressed as median \pm interquartile range (IQR) and were analyzed with the Wilcoxon test. The performance of urinary biomarkers was determined using receiver operating characteristic (ROC) curve analysis. We considered $P<0.05$ to be statistically significant.

\section{Results}

A total of 50 patients were divided into two groups based on the KDIGO criteria, with 11 subjects in the AKI group and 39 in the non-AKI group. The patients' basic and clinical characteristics indicated no significant differences between the two groups, as seen in Table 1. Although there were no differences in $\mathrm{sCr}$ concentrations before surgery, $\mathrm{sCr}$ was higher in the AKI group than in the non-AKI group at 2 and 3 days after surgery (Table 2). Our results indicated a significantly greater need for renal replacement therapy and longer ICU stay in patients in the AKI group as compared to those in the non-AKI group (Table 3). 
Table 1 Patient characteristics

\begin{tabular}{lll}
\hline & $\begin{array}{l}\text { non-AKl group } \\
(n=11)\end{array}$ & $\begin{array}{l}\text { AKI group } \\
(n=39)\end{array}$ \\
\hline Age (years) & $65.2 \pm 11.5$ & $63.8 \pm 13.1$ \\
Sex (male/female) & $4 / 7$ & $16 / 23$ \\
Body weight (kg) & $57.5 \pm 13.6$ & $55.1 \pm 14.3$ \\
Diagnosis & & \\
$\quad$ Coronary artery disease & 2 & 14 \\
$\quad$ Valvular disease & 6 & 20 \\
$\quad$ Ascending aortic aneurysm & 3 & 5 \\
Operating time (min) & $384.1 \pm 67.4$ & $361.9 \pm 69.1$ \\
ACC time (min) & $128.1 \pm 51.4$ & $119.1 \pm 57.4$ \\
CPB time (min) & $162.7 \pm 56.9$ & $157.8 \pm 52.5$ \\
Intraoprerative urine output (ml) & $936 \pm 168.6$ & $1053 \pm 108.6$ \\
Intraoperative fluid balance (min) & $1978 \pm 335.3$ & $2185 \pm 196.1$ \\
\hline
\end{tabular}

There were no significant differences between the two groups $A C C$ aortic closs-clamp, CPB cardio pulmonary bypass

Urinary L-FABP, NGAL, and angiotensinogen were measured at five time points perioperatively. Each marker increased significantly after $\mathrm{CPB}$ compared to baseline levels (before surgery). L-FABP was significantly higher in the AKI group than in the non-AKI group at the end of surgery and $3 \mathrm{~h}$ after surgery. L-FABP levels were $601.5 \pm 341.7$ and $233.8 \pm 127.2 \mu \mathrm{g} / \mathrm{g} \mathrm{Cr}$ in the AKI and non-AKI groups, respectively, at the end of surgery (Fig. 1). Three hours after surgery, NGAL levels were $950.5 \pm 827.9$ and $430.0 \pm 250.6 \mu \mathrm{g} / \mathrm{g} \mathrm{Cr}$ in the AKI and non-AKI groups, respectively, the level being significantly higher in the AKI group than in the non-AKI group for the first time at this point (Fig. 2). In both groups, urinary angiotensinogen levels were the highest at the end of surgery, being $945.3 \pm 522.7$ and $831.0 \pm$ $419.4 \mu \mathrm{g} / \mathrm{g} \mathrm{Cr}$ in the AKI and non-AKI groups, respectively. There were no significant differences in urinary angiotensinogen levels between the two groups at any time point (Fig. 3).

Table 2 Pre- and postoperative serum creatinine ( $\mathrm{s} C r$ ) levels

\begin{tabular}{llll}
\hline $\mathrm{sCr}(\mathrm{mg} / \mathrm{dl})$ & $\begin{array}{l}\text { AKI group } \\
(n=11)\end{array}$ & $\begin{array}{l}\text { non-AKI group } \\
(n=39)\end{array}$ & $P$ value \\
\hline $\begin{array}{l}\text { Preoperative } \\
\text { Postoperative }\end{array}$ & $0.72 \pm 0.14$ & $0.68 \pm 0.15$ & 0.531 \\
$\begin{array}{l}1 \text { day } \\
\text { Postoperative }\end{array}$ & $0.83 \pm 0.11$ & $0.69 \pm 0.16$ & $<0.05$ \\
$\begin{array}{l}\text { days } \\
\text { Postoperative }\end{array}$ & $1.04 \pm 0.15$ & $0.78 \pm 0.19$ & $<0.01$ \\
3 days & $1.05 \pm 0.12$ & $0.77 \pm 0.18$ & $<0.01$ \\
\hline
\end{tabular}

Table 3 Clinical outcomes

\begin{tabular}{clll}
\hline & $\begin{array}{l}\text { AKI group } \\
(n=11)\end{array}$ & $\begin{array}{l}\text { non-AKI group } \\
(n=39)\end{array}$ & $P$ value \\
\hline AKI severity $(n)$ & & & \\
Stage 1 & 6 & 0 & \\
Stage 2 & 3 & 0 & $<0.01$ \\
Stage 3 & 2 & 0 & $<0.01$ \\
Need for RRT $(n)$ & 3 & 0 & 0.057 \\
ICU stay (day) & $8.2 \pm 3.5$ & $3.7 \pm 2.4$ & \\
ICU death $(n)$ & 1 & 0 & \\
\hline RRT renal replacent & & 0 & \\
\hline
\end{tabular}

$R R T$ renal replacement therapy, ICU intensive care unit

\section{ROC analysis of urinary L-FABP and NGAL for prediction} of AKI

ROC analysis of L-FABP for the prediction of AKI is shown in Fig. 4a, while Fig. 4b shows ROC analysis of NGAL. These results between the AKI and non-AKI group were analyzed at the most significantly different time points, which were at the end of surgery for LFABP levels and $3 \mathrm{~h}$ after surgery for NGAL levels. The area under the curve (AUC) of NGAL was more than that of L-FABP, although both NGAL and L-FABP were found to be significantly positive predictive markers of AKI. In addition, the cutoff values of L-FABP at the end of surgery and NGAL at $3 \mathrm{~h}$ after surgery for diagnosing AKI, as analyzed from ROC curves, were 357.5 and $670.5 \mu \mathrm{g} / \mathrm{g} \mathrm{Cr}$, respectively.

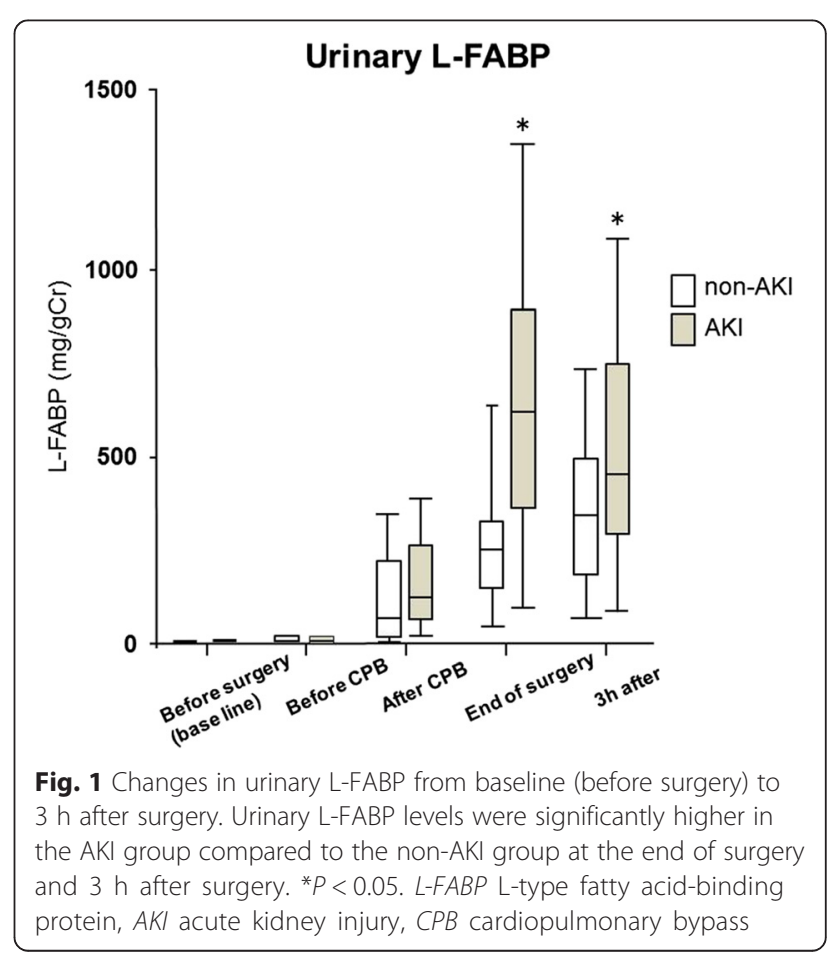




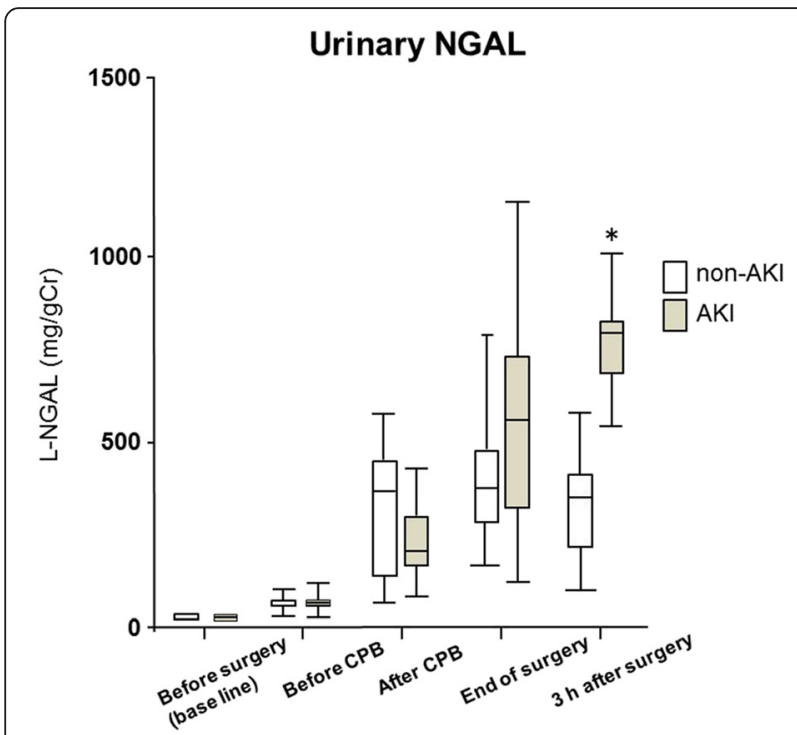

Fig. 2 Changes in urinary NGAL from baseline (before surgery) to $3 \mathrm{~h}$ after surgery. Urinary NGAL levels were significantly higher in the AKI group compared to the non-AKI group $3 \mathrm{~h}$ after surgery. ${ }^{*} P<0.05$. NGAL neutrophil gelatinase-associated lipocalin, AKI acute kidney injury, CPB cardiopulmonary bypass

\section{Discussion}

\section{Key results}

In the present study, of the three biomarkers assessed, L-FABP increased most rapidly in the AKI as compared to the non-AKI group. Moreover, there were significant differences between the AKI and non-AKI group in the levels of L-FABP and NGAL. NGAL was a more efficient

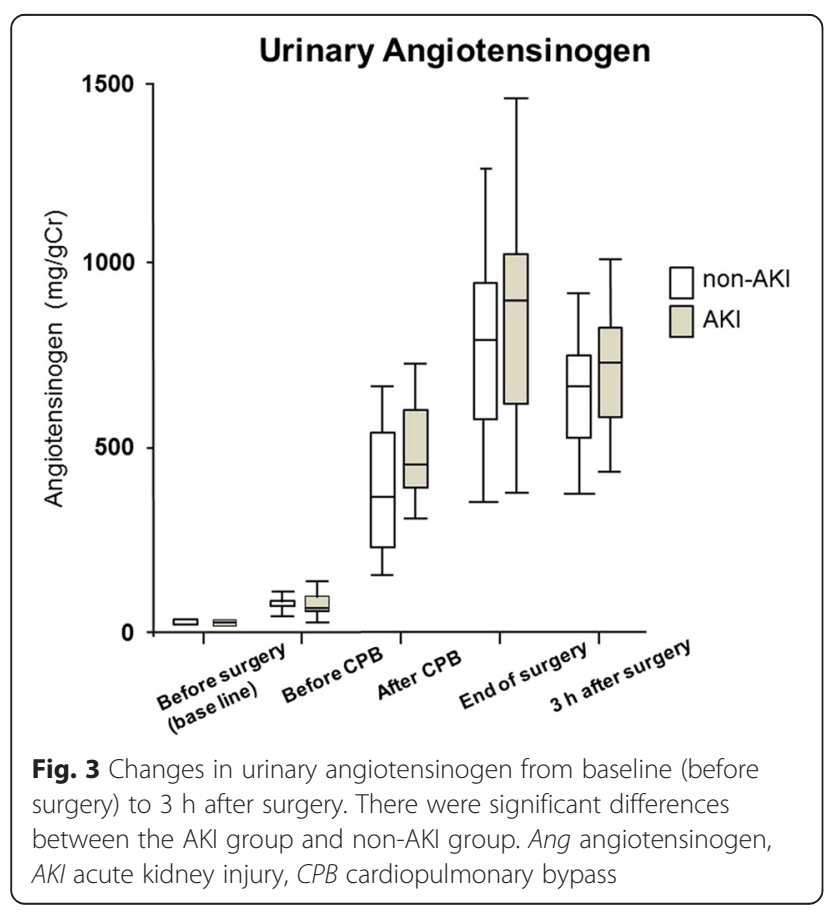

marker for prediction of the incidence of AKI, because the AUC-ROC of NGAL $3 \mathrm{~h}$ after surgery was higher than that of L-FABP at the end of surgery.

\section{Issues with $\mathrm{sCr}$ and the need for new biomarkers for the diagnosis of AKI} $\mathrm{sCr}$ has been identified as the main index of kidney injury, and the diagnosis of AKI is mainly based on $\mathrm{sCr}$ concentrations in the RIFLE, AKIN, and KDIGO criteria [4-6]. Recently, several research groups reported that small increments in $\mathrm{sCr}$ after cardiac surgery, which was not adopted as a diagnostic criterion for AKI, were significantly associated with the progression of postoperative AKI and mortality [3, 11-14]. The underlying mechanism by which a minimal increase in $\mathrm{sCr}$ can indicate worsening progression after surgery is not clear. Further, hemodilution and hydration may lead to falsely low sCr measurements, which in turn can confound the diagnosis of AKI after cardiac surgery. This low accuracy of $\mathrm{sCr}$ measurements, in addition to the delay in the increase in $\mathrm{sCr}$ after cardiac surgery, is another drawback of using $\mathrm{sCr}$ as a marker of AKI. With the early detection of AKI, clinical interventions, such as maintaining adequate renal blood flow and blood pressure, and avoiding the development of hypovolemia, anemia, and unnecessary blood transfusion, can be undertaken, which might contribute to reducing the severity of the renal damage.

Therefore, a rapid and efficient biomarker is much needed for the early diagnosis of postoperative AKI. Until now, there was no ideal and complete biomarker, although numerous biomarkers have been previously investigated.

\section{Urinary L-FABP, NGAL, and angiotensinogen}

In the present study, we investigated three urinary biomarkers, L-FABP, NGAL, and angiotensinogen, which are produced and secreted into urine via different mechanisms. L-FABP is a $14-\mathrm{kDa}$ fatty acid-binding protein and is a biomarker that is secreted in urine as a result of reactive oxygen stress. The usefulness of L-FABP as a biomarker in the early detection of AKI after cardiac surgery has been previously reported $[15,16]$. NGAL is a $25-\mathrm{kDa}$ protein belonging to the lipocalin superfamily, which is secreted by neutrophils and renal tubular cells [17]. Urinary NGAL has also been recognized as an early biomarker of AKI after cardiac surgery $[18,19]$. In the present study, although both urinary NGAL and L-FABP concentrations increased immediately after $\mathrm{CPB}$ in both the AKI and non-AKI groups, the levels of urinary LFABP and NGAL peaked at the end of surgery and $3 \mathrm{~h}$ after surgery, respectively. At these time points, the cutoff values of L-FABP and NGAL for 

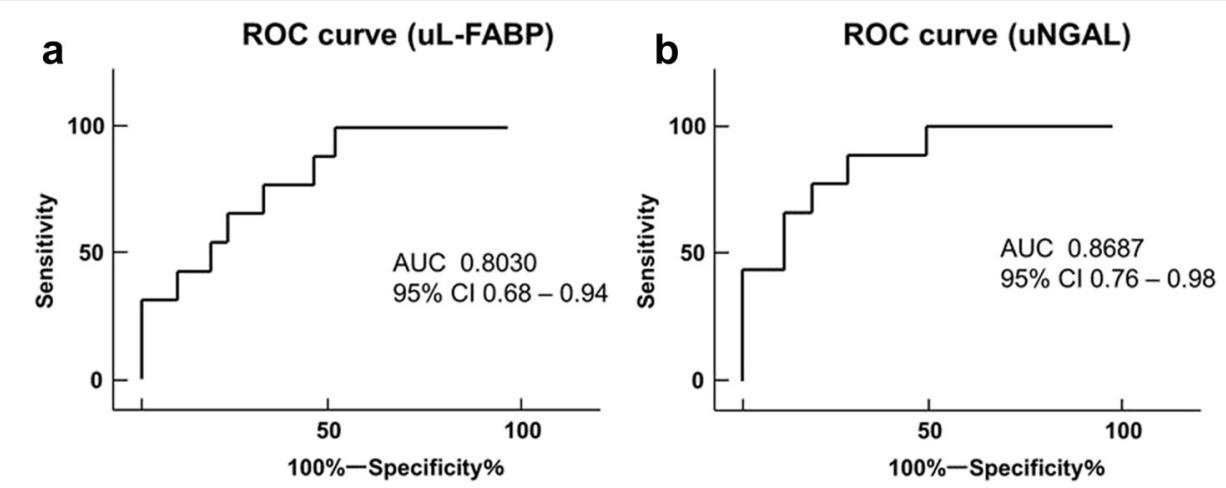

Fig. 4 a ROC curves for AKI based on UL-FABP levels at the end of surgery. The AUC was 0.8030 (95\% Cl 0.68-0.94. ROC receiver operating characteristic, AUC area under the curve, UL-FABP urinary L-type fatty acid-binding protein, AKI acute kidney injury. $\mathbf{b}$ ROC curves for AKI based on uNGAL levels $3 \mathrm{~h}$ after surgery. The AUC was 0.8687 (95\% Cl 0.76-0.98). ROC receiver operating characteristic, AUC area under the curve, UNGAL urinary neutrophil gelatinase-associated lipocalin, AKI acute kidney injury

diagnosing AKI, as analyzed from ROC curves, were 357.5 and $670.5 \mu \mathrm{g} / \mathrm{g} \mathrm{Cr}$, respectively.

There is a difference between the mechanisms of increase in urinary NGAL and L-FABP after CPB. Oxidative stress via renal tissue hypoxia up-regulates L-FABP expression and increases the secretion of L-FABP into urine from damaged proximal tubules [20]. In contrast, inflammation induced by various stresses increases serum NGAL, while impaired renal absorption increases urinary NGAL excretion [21]. Therefore, the increase in urinary NGAL levels takes a longer time as compared with urinary L-FABP levels [22]. On the other hand, urinary angiotensinogen levels were not significantly different between the two groups at all time points. Recently, the existence of independent renin-angiotensinaldosterone systems (RAAS) in several organs and tissues has been reported. All components of the renal RAAS are produced in the kidney, being completely independent of the systemic RAAS, and contribute to the progression of both acute and chronic kidney diseases [23]. Moreover, urinary angiotensinogen has been identified as an index of the activity of the renal RAAS [24], and a previous study showed that elevated urinary angiotensinogen was associated with adverse outcomes after cardiac surgery in patients with AKI [25]. Therefore, urinary angiotensinogen has been suggested to have utility as a prognostic biomarker of AKI after cardiac surgery, similar to other previous biomarkers, including L-FABP and NGAL. Indeed, urinary angiotensinogen levels were significantly increased in both the AKI and non-AKI groups after $\mathrm{CPB}$, as compared to baseline (before the surgery). Although the intrarenal RAAS is activated by $\mathrm{CPB}$, the efficacy of urinary angiotensinogen as a biomarker might not be significant, because the activated intrarenal RAAS is merely one of many factors causing AKI after cardiac surgery. Although the usefulness of several biomarkers for detecting AKI have been previously reported $[16,26]$, in this study, we focused on comparisons between three biomarkers at the same time courses that are produced via different mechanisms. The present results, which confirm the increase in L-FABP and NGAL levels with AKI, suggest that one of the major mechanisms causing AKI after cardiopulmonary bypass might be an increase in reactive oxygen stress, followed by activation of an intrarenal inflammatory reaction.

\section{Limitations}

The present study was conducted at a single institution, and data analysis was not blinded although surgery and anesthesia proceeded normally in all cases. Urinary angiotensinogen concentrations had a wider range as compared to L-FABP and NGAL, suggesting that the sample size might have been too small to assess the utility of these biomarkers for the detection of AKI. A panel of biomarkers would, thus, seem to be more useful for the early detection of AKI [27], although we could not demonstrate the advantage of assessing both L-FABP and NGAL, because the time points at which each of these biomarkers peaked were different. This might also be a limitation of our study.

\section{Conclusions}

We investigated the efficacy of three biomarkers of AKI and demonstrated the utility of L-FABP and NGAL, but not angiotensinogen. All three biomarkers increased after $\mathrm{CPB}$, although they peaked at different time points during the observation period. Hence, although evaluating a panel of biomarkers would probably help overcome the delay in the diagnosis of AKI after cardiac surgery, the problem of the different peak points among biomarkers needs to be resolved for discovery of a panel of biomarkers. 


\section{Availability of supporting data}

The datasets supporting the conclusions of this article are included within the article and its additional file.

\begin{abstract}
Abbreviations
AKI: Acute kidney injury; L-FABP: L-type fatty acid-binding protein; NGAL: Neutrophil gelatinase-associated lipocalin; sCr: Serum creatinine; RIFLE: Risk, Injury, Failure, Loss of kidney function and End stage of kidney disease; AKIN: Acute Kidney Injury Network; GFR: Gomerular filtration rate; CPB: Cardiopulmonary bypass; BIS: Bispectral index; KDIGO: Kidney Disease: Improving Global Outcomes; SD: Standard deviations; ROC: Receiver operating characteristic; RAAS: Renin-angiotensin-aldosterone systems.
\end{abstract}

\section{Competing interests}

The authors declare that they have no competing interests.

\section{Authors' contributions}

TM conceived the study idea and drafted the manuscript. SH, TS, MN, and SI helped with the data collection and data analysis. YK revised and edited the manuscript. All authors read and approved the final manuscript.

\section{Acknowledgements}

There are no additional acknowledgments.

\section{Funding}

None of the authors received any funding for this study.

\section{Author details}

${ }^{1}$ Department of Anesthesiology and Intensive Care, Kagoshima University Hospital, Sakuragaoka 8-35-1, Kagoshima 46201, Japan. ²Department of Anesthesiology and Critical Care Medicine, Kyusyu University, Maidashi 3-1-1, Higashi-ku, Fukuoka 46201, Japan.

Received: 4 March 2016 Accepted: 15 June 2016

Published online: 21 June 2016

\section{References}

1. Gaffney AM, Sladen RN. Acute kidney injury in cardiac surgery. Curr Opin Anaesthesiol. 2015:28:50-9.

2. Thiele RH, Isbell JM, Rosner MH. AKI associated with cardiac surgery. Clin J Am Soc Nephrol. 2015;10:500-14.

3. Lassnigg A, Schmidlin D, Mouhieddine M, Bachmann LM, Druml W, Bauer P et al. Minimal changes of serum creatinine predict prognosis in patients after cardiothoracic surgery: a prospective cohort study. J Am Soc Nephrol. 2004;15:1597-605.

4. Bellomo R, Ronco C, Kellum JA, Mehta RL, Palevsky P. Acute Dialysis Quality Initiative work group. Acute renal failure-definition, outcome measures, animal models, fluid therapy and information technology needs: the Second International Consensus Conference of the Acute Dialysis Quality initiative (ADQI) Group. Crit Care. 2004;8:R204-8.

5. Mehta RL, Kellum JA, Shah SV, Molitoris BA, Ronco C, Wamock DG, et al. Acute Kidney Injury Network: report of an initiative to improve outcomes in acute kidney injury. Crit Care. 2007;11:R31.

6. Kidney Disease: Improving Global Outcomes (KDIGO) practice guideline for acute kidney injury. Kidney Int Suppl. 2012;2:1-138.

7. Kellen M, Aronson S, Roizen MF, Barnard J, Thisted RA. Predictive and diagnostic tests of renal failure: a review. Anesth Analg. 1994;78:134-42.

8. Najafi M, Goodarzynejad H, Karimi A, Ghisai A, Soltaninia H, Marzban M, et al. Is preoperative serum creatinine a reliable indicator of outcome in patients undergoing coronary artery bypass surgery. J Thorac Cardio Vasc Surg. 2009:137:304-8.

9. Wijeysundera DN, Karkouti K, Beattie WS, Rao V, Ivanov J. Improving the identification of patients at risk of postoperative renal failure after cardiac surgery. Anesthesiology. 2009;104:65-72.

10. Swedko PJ, Clark HD, Paramsothy K, Akbari A. Serum creatinine is an inadequate screening test for renal failure in elderly patients. Arch Intern Med. 2003;163:356-60.

11. Lassnig A, Schmid ER, Hiesmayr M, Falk C, Druml W, Bauer P, et al. Impact of minimal increases in serum creatinine on outcome in patients after cardiothoracic surgery: do we have to revise current definitions of acute renal failure? Crit Care. 2008;36:1129-37.
12. Ishani A, Nelson D, Clothier B, Schult T, Nugent S, Greer N, et al. The magnitude of acute serum creatinine increase after cardiac surgery and the risk of chronic kidney diseases, progression of kidney disease, and death. Arch Intern Med. 2011;1171:226-33.

13. Tolpin DA, Collard CD, Lee W, Virani SS, Allison PM, Elayda MA, et al. Subclinical changes in serum creatinine and mortality after bypass grafting. J Thorac Cardiovasc Surg. 2012;143:682-8.

14. Liotta M, Olsson D, Sartipy U, Holzmann MJ. Minimal changes in postoperative creatinine values and early and late mortality and cardiovascular events after coronary artery bypass grafting. Am J Cardiol. 2014;113:70-5.

15. Portilla D, Dent C, Sugaya T, Nagothu KK, Kund I, Moore P, et al. Liver fatty acid-binding protein as a biomarker of acute kidney injury after cardiac surgery. Kidney Int. 2008;73:465-72.

16. Matsui K, Kamijo-Ikemori A, Sugaya T, Yasuda T, Kimura K. Usefulness of urinary biomarkers in early detection of acute kidney injury after cardiac surgery in adults. Circ J. 2012;76:213-20.

17. Mishra J, Ma Q, Prada A, Mitsnefes M, Zahedi K, Yang J, et al. Identification of neutrophil gelatinase-associated lipocalin as a novel early urinary biomarker for ischemic renal injury. J Am Soc Nephrol. 2003;14:2534-43.

18. Mishra J, Dent C, Tarabishi R, Mitsnefes MM, Ma Q, Kelly C, et al. Neutrophil gelatinase-associated lipocalin (NGAL) as a biomarker for acute renal injury after cardiac surgery. Lancet. 2005;365:1231-38.

19. Haase M, Bellomo R, Devarajan P, Schlattmann P, Haase-Fielitz A, NGAL Meta-analysis Investigator Group. Accuracy of neutrophil gelatinaseassociated lipocalin (NGAL) in diagnosis and prognosis in acute kidney injury: a systematic review and meta-analysis. Am J Kidney Dis. 2009;54: 1012-24.

20. Matsui K, Kamijo-Ikemorif A, Sugaya T, Yasuda T, Kimura K. Renal liver-type fatty acid binding protein (L-FABP) attenuates acute kidney injury in aristolochic acid nephrotoxicity. Am J Pathol. 2011;178:1021-32.

21. Kuwabara T, Mori K, Mukoyama M, Kasahara M, Yokoi H, Saito Y, et al. Urinary neutrophil gelatinase-associated lipocalin levels reflect damage to glomeruli, proximal tubules, and distal nephrons. Kidney Int. 2009;75:285-94.

22. Efrati S, Berman S, Abu Hamad R, El Nakib R, Chanimov M, Siman-Tov Y, et al. Hyperglycaemia, inflammation, RAS activation: three culprits to blame for acute kidney injury emerging in healthy rats during general anesthesia. Nephrology. 2012;17:591-602.

23. Kobori H, Nangaku M, Navar LG, Nishiyama A. The intrarenal renin-angiotensin system: from physiology to the pathobiology of hypertension and kidney disease. Pharmacol Rev. 2007;59:251-87.

24. Kobori H, Alper Jr AB, Shenava R, Katsurada A, Saito T, Ohashi N, et al. Urinary angiotensinogen as a novel biomarker of the intrarenal renin-angiotensin system status in hypertensive patients. Hypertension. 2009;53:344-50.

25. Alge $\mathrm{L}$, Karakata N, Neely BA, Janech MG, Tumlin JA, Chawla LS, et al. Urinary angiotensinogen and risk of severe AKI. Clin J Am Soc Nephrol. 2013;8:184-93.

26. Arthur JM, Hill EG, Alge JL, Lewis EC, Neely BA, Janech MG, et al. Evaluation of 32 urine biomarkers to predict the progression of acute kidney injury after cardiac surgery. Kidney Int. 2014;85:431-8.

27. Wyckoff T, Augoustides JG. Advances in acute kidney injury associated with cardiac surgery: the unfolding revolution in early detection. J Cardiothorac Vasc Anesth. 2012;26:340-5.

\section{Submit your next manuscript to BioMed Central} and we will help you at every step:

- We accept pre-submission inquiries

- Our selector tool helps you to find the most relevant journal

- We provide round the clock customer support

- Convenient online submission

- Thorough peer review

- Inclusion in PubMed and all major indexing services

- Maximum visibility for your research

Submit your manuscript at www.biomedcentral.com/submit 